\title{
Dyslipidemia in Chinese Pancreatic Cancer Patients: A Two-Center Retrospective Study
}

Feiyang Wang ( $\sim 1335528702 @ q q . c o m)$

Shanghai General Hospital https://orcid.org/0000-0002-1457-0077

Heshui Wu

Wuhan Xiehe Hospital: Wuhan Union Hospital

Junming $X u$

Shanghai General Hospital

\section{Research article}

Keywords: Dyslipidemia, pancreatic cancer

Posted Date: April 9th, 2021

DOI: https://doi.org/10.21203/rs.3.rs-386978/v1

License: () (i) This work is licensed under a Creative Commons Attribution 4.0 International License. Read Full License 


\section{Abstract}

Background: Pancreatic cancer (PC) is one of the most aggressive and lethal malignancies in the world. High cholesterol intake may have a certain association with an elevated risk of PC, though dyslipidemia in PC patients has rarely been reported. In this study, we compared serum lipids levels between $\mathrm{PC}$ and non-PC tumor patients and assessed their prognostic value in PC.

Methods: 271 patients treated at Wuhan Union Hospital from January 2012 to December 2016 and 204 individuals at Shanghai General Hospital from January 2018 to December 2019 were recruited. Their demographic parameters, laboratory data, pathological information, and clinical outcomes were extracted and analyzed. The mRNA expressions of related lipoprotein, low density lipoprotein receptor (LDLR) and high density lipoprotein binding protein (HDLBP), in PC tissues and paired noncancerous tissues and follow-up information were assessed based on the GEO database (GSE15471 and GSE62165) and TCGA database.

Results: A total of 172 non-PC tumor patients and 260 PC patients were finally eligible for our analysis. PC patients exhibited higher levels of serum triglyceride, cholesterol, and low-density lipoprotein (LDL) and a lower serum high-density lipoprotein (HDL) level on admission versus the non-PC tumor group. In PC patients, LDLR mRNA expression was upregulated, and HDLBP mRNA expression was downregulated in cancerous tissues compared to these levels in paired noncancerous tissues. The survival analysis revealed that dyslipidemia had a non-significant association with a poor prognosis, but PC patients with a high LDLR level were at risk of poor survival.

Conclusion: Dyslipidemia is detected in PC patients but has a non-significant relation to PC prognosis. However, LDLR may be a potential predictive marker for PC prognosis.

\section{Introduction}

Pancreatic cancer (PC) is the ninth common malignant tumor worldwide. It has a dismal 5-year survival rate of less than $9 \%$, which is expected to be the second deadliest cancer by 2030. Disappointingly, $80 \%$ of patients have developed regional or distant metastasis at first diagnosis [1].

With rapid advances in the diagnostic efficiency of imaging and laboratory techniques, there is an increased detection rate of PC over the last decades. But, some types of non-pancreatic cancer (non-PC) tumor, including pancreatic neuroendocrine tumor (PNET), intraductal papillary mucinous neoplasms (IPMNs), and pancreatic cystadenoma are still difficult to distinguish from PC without pathological means. Recently, research have found high cholesterol intake is associated with an elevated risk of PC [2-3], and lipid metabolism reprogramming is the key factor promoting PC development and chemotherapy resistance [4-5]. Some lipoproteins, such as ApoE and ApoA-II, are recognized as potential biomarkers with higher sensitivity than current serum indicators for PC [2, 6]. All these findings point directly at functional changes in lipid metabolism of PC. But by far, deviant serum lipid levels in PC and differences between PC and nonPC tumor patients have been rarely remarked.

In this work, we compared baseline serum lipid levels between PC versus non-PC tumor patients and examined their diagnostic utility in distinguishing PC from non-PC tumors. The prognostic potential of serum lipid levels in PC patients was also assessed. To better understand the role of lipid metabolism in the pathogenesis, we utilized GEO and TCGA data to identify differential targets between PC and non-PC tumors and evaluate their prognostic value in PC. This study provides fresh insights into lipid metabolism in PC development and progression.

\section{Materials And Methods}

\section{Patients and data collection}

We extracted the medical records of 271 patients hospitalized and undergoing surgical resection in Wuhan Union Hospital from January 2012 to December 2016 and 204 patients in Shanghai General Hospital from January 2018 to December 2019. Among them, 260 patients were pathologically confirmed as PC and 172 patients as non-PC tumor types (PNET, IPMN, chronic pancreatitis, solid pseudopapillary tumor, pancreatic cystadenoma, and other types). 43 patients without complete hospitalization or follow-up data were excluded (Figure 1). Demographic characteristics, laboratory results, serum lipids levels, surgical and pathological data were collected through electronic medical records and paper charts. Patient outcomes were evaluated based on outpatient records and telephone interviews. The follow-up was ended on March 7, 2020.

\section{Microarray data collection}

Data sets of gene expressions used in this study were downloaded from the GEO database (http://www.ncbi.nlm,nih.gov/gds/) and derived from microarray studies comparing the mRNA expression profiling between PC and paired adjacent noncancerous tissues from patients. The mRNA expression data of GSE15471 (39 matched pairs of samples) and GSE62165 (118 PC tissue samples and 13 paracancerous tissue samples) were acquired to identify differential target genes of related lipoprotein in PC. Follow up information of PC patients were obtained from the Cancer Genome Atlas (TCGA) data portal (http://cancergenome.nih.gov/). According to the expression of related lipoprotein, a total of 177 PC patients with follow up information were selected for survival analysis .

\section{Statistical analysis}

Statistical Production and Services Solution 19.0 (SPSS 19.0, SPSS Inc, Chicago, IL, USA) and GraphPad Prism 7 were used for statistical analysis. Continuous and categorical variables were presented as mean \pm standard derivation (SD), as well as frequency and percentage, respectively. Normally distributed continuous variables were compared by using Student's $t$-test, and differences in categorical variables were analyzed using the chi-square or 
Fisher's exact test. The Mann-Whitney Utest was used for comparisons in nonnormally distributed variables. A logistic regression analysis model was used to identify risk factors of PC. Only the variables with statistical significance in univariable analysis were included in further multivariable analysis. Odds ratios

(ORs) and 95\% confidence intervals (95\% Cls) were presented. Patient disease-specific survival (DSS) was obtained using the Kaplan-Meier method. A P-value $<0.05$ was considered a statistically significant difference.

\section{Results}

\section{Clinical features, laboratory data, and serum lipids levels between PC versus non-PC tumors}

A total of 432 patients undergoing surgical resection at the two centers from January 2012 to December 2019 were included in this study. Among them, 260 patients were diagnosed as PC and 172 patients as non-PC tumors pathologically. Their demographic and clinical characteristics, as well as laboratory information, were summarized in Table 1. There were 167 males (67.5\%; median age, 61 years; range, 23-94 years) in the PC group and 87 males (50.6\%; median age, 55 years; range, 16-82 years) in the non-PC tumor group. Compared with non-PC tumor patients, PC patients showed significant differences in age $(P<0.001)$, gender $(P=0.005)$, and symptomatology $(P<0.001)$ at baseline. Elder age, male gender, alcohol intake $(P=0.011)$, and the first symptoms as abdominal pain and jaundice could be risk factors for PC. Among laboratory indicators, $P C$ patients exhibited lower hemoglobin ( $P=0.002)$, lower albumin level $(P<0.001)$ and higher levels of total bilirubin $(P<0.001)$, alanine aminotransferase $(P<0.001)$, serum glucose $(P<0.001)$, and $C A 199(P<0.001)$ in serum. Besides, activated partial thromboplastin times were significantly prolonged in the $P C$ group $(P=0.02)$ versus patients with non-PC tumors. As shown in Table 1 and Figure 2, $P C$ patients presented a serum lipids difference from non- $P C$ tumor patients in triglyceride $(P=0.001)$, cholesterol $(P=0.005), H D L(P<0.001)$, $\operatorname{LDL}(\mathrm{P}=0.003)$, apolipoprotein $\mathrm{A} 1(\mathrm{P}=0.001)$, apolipoprotein $\mathrm{B}(\mathrm{P}=0.007)$, and apolipoprotein $\mathrm{E}(\mathrm{P}<0.001)$.

\section{Logistic regression analysis of risk factors for PC}

The univariable logistic regression analysis revealed that male gender $(P=0.005)$, elder age $(P=0.002)$, alcohol intake $(P=0.012)$, lower hemoglobin $(0.002)$, higher levels of total bilirubin $(P<0.001)$, alanine aminotransferase $(P<0.001)$, serum glucose $(P=0.001)$, and CA199 $(P<0.001)$, together with dyslipidemia (triglyceride: $\mathrm{P}=0.007$, cholesterol: $\mathrm{P}=0.003, \mathrm{HDL}: \mathrm{P}=0.012$, and $\mathrm{LDL}: \mathrm{P}=0.015$ ), were significantly correlated with $\mathrm{PC}$. These characteristics above were further screened using the multivariable analysis. As shown in Table 2, only increased total bilirubin $(P=0.004)$, alanine aminotransferase $(P=0.002)$, and $C A 199$ $(P<0.001)$ levels on admission were the independent risk factors of PC.

\section{Clinicopathological features and survival analysis between PC patients with different lipid levels}

As listed in Table 3, clinicopathological features and prognostic data of PC patients were analyzed based on different lipid level. We found cholesterol $(P<0.001)$ and LDL $(P<0.001)$ levels were related to tumor size. $P C$ patients with a high LDL level had longer follow-up $(P=0.009)$ because of the within-group difference in number of cases. According to an LDL cutoff of $4.14 \mathrm{mmol} / \mathrm{L}, 27 \mathrm{PC}$ patients were assigned to the high-LDL group versus 201 patients in the low-LDL group. Besides, there were non-significant differences in other indicators, including tumor stage, degree of differentiation, nerve and vascular invasion, between the two groups. The Kaplan-Meier survival analysis showed that dyslipidemia in PC patients had no significant correlation with DSS (Figure 3), while patients with a low HDL level tended to have a poor prognosis $(P=0.183)$.

\section{Differential expression of LDLR and HDLBP mRNAs and their prognostic value in PC}

LDLR is a cell-surface receptor that metabolizes elevated levels of plasma LDL-cholesterol (LDL-C) to regulate cholesterol homeostasis in the circulation [7]. HDLBP is a protein that specifically binds to HDL molecules and may function in the removal of excess cellular cholesterol [8]. We analyzed the mRNA expression levels of LDLR and HDLBP using GSE15471 and GSE62165 cohorts from GEO. The results showed that LDLR (GSE15471: P=0.044; GSE62165: $\mathrm{P}<0.001)$ mRNA expression significantly increased in PC tissues, and HDLBP (GSE15471: $P<0.001$; GSE62165: $P<0.001$ ) mRNA expression were downregulated compared with the expressions in paired adjacent noncancerous tissues. Subsequently, differences in clinical features between patients with different LDLR and HDLBP mRNA expression levels were compared using the data from 177 PC patients in TCGA database. The basic characteristics and follow-up information were presented in Table S1. The results showed that PC patients with a high LDLR expression level were associated with a poor prognosis $(P=0.003)$ (Figure 4$)$, and patients with both high LDLR $(P=0.001)$ and HDLBP $(P=0.042)$ expressions exhibited a higher incidence of cancer-related death versus those with low LDLR and HDLBP expressions (Table S2).

\section{Discussion}

Metabolic reprogramming has been ascertained to be a hallmark of cancer, of which abnormal glucose and lipid metabolism are key players promoting the progression of PC $[5,9]$. However, differences in baseline serum lipid levels between PC and non-PC tumor types are rarely noticed. Our results demonstrated these disparities in baseline clinical features and laboratory parameters between the two groups. Specifically, PC patients with dyslipidemia had higher serum triglyceride, cholesterol, and LDL levels and a decreased HDL level. Compared to common diagnostic factor for PC, CA199 for example, these lipid indicators did not show a notable role in diagnosis and prognosis evaluation of PC. Regarding lipid-related mRNA markers LDLR and HDLBP (the cellular receptors for LDL and HDL), they also displayed a potential diagnostic performance, showing differential expressions between PC and paracancerous tissues. And PC patients with a high LDLR level significantly correlated with a poor prognosis, as indicated by the TCGA survival analysis.

In the results of our study, lipid regulation in PC is more conducive to elevating serum lipid levels compared to other non-PC tumor types. Some pieces of verifiable evidence support that high dietary cholesterol is associated with an increased risk of PC [2-3]. Food-derived fatty acids are required for the synthesis of phospholipids to in cell proliferation and lipid metabolism signaling [10-11]. Because lipids such as phospholipid bilayers are fundamental structural components enabling cell division [6]. So high serum fat levels contribute to PC development. Meanwhile, to adapt the nutritional needs increase to withstand 
hypoperfusion and hypoxia caused by high interstitial fluid pressures and poor stroma perfusion in the tumor environment, PC cells often manage to survive and develop by fueling lipid transport and metabolism [12-13]. Besides, enzyme activities in lipid signaling are also strengthened. The expressions of lipogenic enzymes participating in the lipid metabolism, such as fatty acid synthase (FASN) and ATP citrate lyase (ACLY), are markedly upregulated in PC patients with poor survival by enhancing chemotherapy resistance [14-15]. These findings suggested how PC cells use the regulation within the lipid microenvironment to promote PC progression. High lipid intake promotes pancreatic carcinogenesis, and PC cells induce cancer development by aberrant lipid metabolism activity.

LDLR is a transcriptional target of SREBP-2. It is also the LDL receptor that functions in increasing serum cholesterol levels in cells through receptor-mediated endocytosis [16]. A higher LDLR expression level is associated with an increased risk of PC recurrence. After LDLR silencing, cancer cell proliferation can be significantly suppressed with enhanced gemcitabine sensitivity [15]. Cholesterol uptake disruption via inactivating LDLR is warranted to develop a novel approach of PC metabolic targeting $[9,17]$. HDLBP, also known as vigilin, was found overexpressed in PC tissues in our study. However, evidence about the relationship between HDLBP and PC is limited. In other cancer types, hepatocellular carcinoma, for example, HDLBP is proved to act as an anti-apoptotic effect in promoting cell proliferation and tumor growth [18]. Since we ascertained the downregulation of HDLBP mRNA expression in this work, whether HDLBP involves in PC onset and development needs further research.

New progress has confirmed cholesterol uptake and lipid metabolism as a highly attractive target for PC metabolic therapy. Statins, inhibitors of HMG-CoA reductase, have been studied in vitro using various cancer cell lines. Antitumor effects of lipophilic statins in PC result mainly from the suppression of proliferation and promotion of apoptosis [19-20]. The roles of statins in improving PC survival also have been reported in some clinical trials [21-22]. Orlistat, a FASN inhibitor, induces endoplasmic reticulum stress and increases gemcitabine sensitivity in mouse models with orthotopic PC implantation [23].

In summary, our study elaborated disparities in dyslipidemia between PC and non-PC tumor patients, but the underlying mechanism has not been fully explored. The roles of specific lipoproteins in PC and their diagnostic and prognostic values were verified. Moreover, key proteins involving lipid metabolic regulation, especially LDLR, are potential prognostic targets for PC. As well, lipid metabolic regulation provides a potential therapy for PC in clinical applications.

\section{Conclusion}

This study highlights dyslipidemia in PC patients and its difference between PC and non-PC tumor types but differentially expressed lipid indicators exhibit no obvious correlations with PC prognosis. Among the related lipoprotein, LDLR is a potential predictive marker for PC prognosis. A new view to distinguish PC from other pancreatic tumors is raised by aberrant blood lipid levels. Our study underlies further PC research on lipid metabolism and the application of lipidregulating drugs in PC therapy. There were still several limitations in this study. As a retrospective study, the dyslipidemia in PC requires to be investigated in a prospective study, and the mechanism of how dyslipidemia affects PC onset and progression requires validations. Negative results of these lipid indicators in diagnostic and survival analysis may be related to the small size and short follow-up periods. Roles of LDLR and HDLBP in PC need to be further verified in experimental research. Nevertheless, this two-center retrospective study, with certain reliability, shed new light on lipid metabolism in PC.

\section{Declarations}

\section{Ethics approval and consent to participate}

The Shanghai General Hospital ethics board approved this study. Patients included in this study consented to their medical records for analysis.

\section{Consent for publication}

All authors consent for publication.

\section{Competing interests}

The authors declare that they have no competing interests.

\section{Funding}

No funding was obtained for this study.

\section{Authors' contributions}

FY and JM designed the study; FY and HS did the data collection and article search; FY analyzed the data and wrote the main manuscript text; all authors have read and approved the manuscript.

\section{Acknowledgements}

The authors thank the staff from Hepatobiliary and Pancreatic Surgery of Shanghai General Hospital for their support during the study. We sincerely appreciated the data provided by Pancreas Surgery of Wuhan Union Hospital.

\section{Abbreviations}


Pnet Pancreatic neuroendocrine tumor

IPMN Intraductal papillary mucinous neoplasms

CP Chronic pancreatitis

SPT Solid pseudopapillary tumor.

TG Triglyceride

CHOL Cholesterol

HDL High density lipoprotein

LDL Low density lipoprotein

GEO Gene Expression Omnibus

TCGA The Cancer Genome Atlas

HDLBP High density lipoprotein binding protein

LDLR Low density lipoprotein receptor

CA199 Caner antigen 199

AJCC American Joint Committee on Cancer

\section{References}

[1] Siegel RL, Miller KD, Jemal A. Cancer statistics, 2020. CA Cancer J Clin. 2020; 70(1): 7-30.

[2] Chen H, Qin S, Wang M, et al. Association between cholesterol intake and pancreatic cancer risk: evidence from a meta-analysis. Sci Rep. 2015 ; 5: 8243.

[3] Wang J, Wang WJ, Zhai L, et al. Association of cholesterol with risk of pancreatic cancer: a meta-analysis. World J Gastroenterol. 2015; 21(12): 3711-3719.

[4] Hanahan, D.; Weinberg, R.A. Hallmark of cancer: The next generation. Cell. 2011; 144(5): 646-674.

[5] Qin C, Yang G, Yang JS, et al. Metabolism of Pancreatic Cancer: Paving the Way to Better Anticancer Strategies. Mol Cancer. 2020; 19(1): 50.

[6] Philip B, Roland CL, Daniluk J, et al. A high-fat diet activates oncogenic Kras and COX2 to induce development of pancreatic ductal adenocarcinoma in mice. Gastroenterology. 2013; 145(6): 1449-1458.

[7] Zhong S, Li L, Zhang YL, et al. Acetaldehyde dehydrogenase 2 interaction with LDLR and AMPK regulate foam formation. J Clin Invest. 2019; 129(1): 252267.

[8] Xia YR, Klisak I, Sparkes RS, et al. Localization of the gene for high-density lipoprotein binding protein (HDLBP) to human chromosome 2q37. Genomics. 1993; 16(2): 524-525.

[9] Romain Cohen, Cindy Neuzillet, Annemilai Tijeras-Raballand, et al. Targeting cancer cell metabolism in pancreatic adenocarcinoma. Oncotarget. 2015; 6(19): 16832-16847.

[10] Louie SM, Roberts LS, Mulvihill MM, et al. Cancer cells incorporate and remodel exogenous palmitate into structural and oncogenic signaling lipids. Biochim Biophys Acta 2013; 1831: 1566-1572.

[11] Kuemmerle NB, Rysman E, Lombardo PS, et al. Lipoprotein lipase links dietary fat to solid tumor cell proliferation. Mol Cancer Ther. 2011; 10 : 427-436.

[12] Provenzano PP, Cuevas $C$, Chang AE, et al. Enzymatic targeting of the stroma ablates physical barriers to treatment of pancreatic ductal adenocarcinoma. Cancer Cell. 2012; 21(3):418-429.

[13] Hidalgo M. Pancreatic cancer. N Engl J Med. 2010; 362(17):1605-1617.

[14] Tadros S, Shukla SK, King RJ, et al. De Novo Lipid Synthesis Facilitates Gemcitabine Resistance through Enoplasmic Reticulum Stress in Pancreatic Cancer. Cancer Res. 2017; 77(20): 5503-5517.

[15] Guillaumond F, Bidaut G, Ouaissi M, et al. Cholesterol uptake disruption, in association with chemotherapy, is a promising combined metabolic therapy for pancreatic adenocarcinoma. Proc Natl Acad Sci U S A. 2015; 112: 2473-2478.

[16] Horton JD, Goldstein JL, Brown MS, et al. SREBPs: Activators of the complete program of cholesterol and fatty acid synthesis in the liver. J Clin Investig. 2002; 109: 1125-1131. 
[17] Yoshiaki Sunami, Artur Rebelo and Jorg Kleeff. Lipid Metabolism and Lipid Droplets in Pancreatic Cancer and Stellate Cells. Cancers (Basel). 2017; 10(1).

[18] Wen LY, Ling W, Wen QH, et al. Vigilin is overexpressed in hepatocellular carcinoma and is required for HCC cell proliferation and tumor growth. Oncol Rep. 2014; 31(5): 2328-2334.

[19] Mistafa O, Stenius U. Statins inhibit Akt/PKB signaling via P2X7 receptor in pancreatic cancer cells. Biochem Pharmacol. $2009 ; 78: 1115-1126$.

[20] Liao J, Chung YT, Yang AL, et al. Atorvastatin inhibits pancreatic carcinogenesis and increases survival in LSL-KrasG12D-LSL-Trp53R172H-Pdx1-Cre mice. Mol Carcinog. 2013; 52: 739-750.

[21] Huang BZ, Chang JI, Li E, et al. Influence of statins and cholesterol on mortality among patients with pancreatic cancer. J Natl Cancer Inst. 2017 ; $109: 2$.

[22] Osmak M. Statins and cancer: current and future prospects. Cancer Lett 2012; 324: 1-12.

[23] Tadros S, Shukla SK, King RJ, et al. De novo lipid synthesis facilitates gemcitabine resistance through endoplasmic reticulum stress in pancreatic cancer. Cancer Res. 2017; 77: 5503-5517.

\section{Tables}

Table 1 Demographic characteristics and laboratory data of patients with PC and non-PC tumor 


\begin{tabular}{|c|c|c|c|c|c|}
\hline Characteristics & & Pancreatic cancer & & non-PC tumor & P-value \\
\hline Age, years & $\Sigma 260$ & $60.48 \pm 10.77$ & $\Sigma 172$ & $53.29 \pm 14.41$ & $<0.001$ \\
\hline Gender, male & $\Sigma 260$ & $167(64.2 \%)$ & $\Sigma 172$ & $87(50.6 \%)$ & 0.005 \\
\hline Smoke & $\Sigma 260$ & $62(23.8 \%)$ & $\Sigma 172$ & $34(19.8 \%)$ & 0.319 \\
\hline Alcohol & $\Sigma 260$ & $51(19.6 \%)$ & $\Sigma 172$ & $18(10.5 \%)$ & 0.011 \\
\hline $\mathrm{BMl}$ index, kg/m2 & $\Sigma 260$ & $22.17 \pm 3.23$ & $\Sigma 172$ & $22.32 \pm 3.03$ & 0.639 \\
\hline Comorbidities & & & & & 0.545 \\
\hline Circulatory system diseases & $\Sigma 260$ & $69(26.5 \%)$ & $\Sigma 172$ & $41(23.8 \%)$ & \\
\hline Metablolic diseases & $\Sigma 260$ & $53(20.4 \%)$ & $\Sigma 172$ & $24(14.0 \%)$ & \\
\hline Chornic inflammation & $\Sigma 260$ & $18(6.9 \%)$ & $\Sigma 172$ & $17(9.9 \%)$ & \\
\hline Other malignant tumor & $\Sigma 260$ & $8(3.1 \%)$ & $\Sigma 172$ & $4(2.3 \%)$ & \\
\hline Symptom & & & & & $<0.001$ \\
\hline Abdominal pain & $\Sigma 260$ & $146(56.2 \%)$ & $\Sigma 172$ & $60(34.9 \%)$ & \\
\hline Jaundice & $\Sigma 260$ & $95(36.5 \%)$ & $\Sigma 172$ & $8(4.7 \%)$ & \\
\hline Asymptomatic & $\Sigma 260$ & $31(11.9 \%)$ & $\Sigma 172$ & $90(52.3 \%)$ & \\
\hline Others & $\Sigma 260$ & $18(6.9 \%)$ & $\Sigma 172$ & $17(9.9 \%)$ & \\
\hline \multicolumn{6}{|l|}{ Laboratory data } \\
\hline White cell count, $x 109 / L$ & $\Sigma 255$ & $5.62 \pm 1.62$ & $\Sigma 172$ & $5.86 \pm 1.93$ & 0.168 \\
\hline Hemoglobin, g/L & $\Sigma 255$ & $123.97 \pm 17.20$ & $\Sigma 172$ & $129.39 \pm 17.58$ & 0.002 \\
\hline Platelet count, $x 109 / \mathrm{L}$ & $\Sigma 255$ & $214.12 \pm 74.61$ & $\Sigma 172$ & $208.09 \pm 75.13$ & 0.414 \\
\hline Total bilirubin, $\mu \mathrm{mol} / \mathrm{L}$ & $\Sigma 255$ & $102.54 \pm 118.77$ & $\Sigma 171$ & $22.38 \pm 49.9$ & $<0.001$ \\
\hline Alanine aminotransferase, $\mathrm{U} / \mathrm{L}$ & $\Sigma 254$ & $140.89 \pm 176.78$ & $\Sigma 171$ & $42.90 \pm 85.03$ & $<0.001$ \\
\hline Albumin, g/L & $\Sigma 255$ & $39.21 \pm 5.21$ & $\Sigma 170$ & $41.05 \pm 5.24$ & $<0.001$ \\
\hline Creatinine, $\mu \mathrm{mol} / \mathrm{L}$ & $\Sigma 255$ & $62.38 \pm 15.50$ & $\Sigma 171$ & $61.68 \pm 16.38$ & 0.657 \\
\hline Serum glucose, $\mathrm{mmol} / \mathrm{L}$ & $\Sigma 250$ & $6.72 \pm 2.69$ & $\Sigma 165$ & $5.61 \pm 1.90$ & $<0.001$ \\
\hline Prothrombin time, s & $\Sigma 252$ & $12.38 \pm 1.54$ & $\Sigma 170$ & $12.12 \pm 1.54$ & 0.085 \\
\hline Activated partial thromboplastin time, $s$ & $\Sigma 251$ & $33.91 \pm 6.80$ & $\Sigma 169$ & $32.36 \pm 6.41$ & 0.020 \\
\hline CA199, U/ml & $\Sigma 238$ & $450.23 \pm 493.76$ & $\Sigma 163$ & $32.19 \pm 107.84$ & $<0.001$ \\
\hline \multicolumn{6}{|l|}{ Serum lipids level } \\
\hline Triglyceride, $\mathrm{mmol} / \mathrm{L}$ & $\Sigma 242$ & $1.82 \pm 1.37$ & $\Sigma 157$ & $1.41 \pm 1.01$ & 0.001 \\
\hline Cholesterol, $\mathrm{mmol} / \mathrm{L}$ & $\Sigma 242$ & $4.93 \pm 2.35$ & $\Sigma 158$ & $4.36 \pm 1.18$ & 0.005 \\
\hline High density lipoprotein, mmol/L & $\Sigma 231$ & $0.98 \pm 0.49$ & $\Sigma 147$ & $1.17 \pm 0.39$ & $<0.001$ \\
\hline Low density lipoprotein, $\mathrm{mmol} / \mathrm{L}$ & $\Sigma 229$ & $2.97 \pm 1.67$ & $\Sigma 146$ & $2.50 \pm 1.09$ & 0.003 \\
\hline lipoprotein a, mmol/L & $\Sigma 82$ & $207.22 \pm 361.98$ & $\Sigma 80$ & $264.99 \pm 444.73$ & 0.365 \\
\hline Apolipoprotein A1, g/L & $\Sigma 82$ & $1.11 \pm 0.38$ & $\Sigma 81$ & $1.29 \pm 0.29$ & 0.001 \\
\hline Apolipoprotein B, g/L & $\Sigma 82$ & $0.88 \pm 0.35$ & $\Sigma 81$ & $0.76 \pm 0.22$ & 0.007 \\
\hline Apolipoprotein E, mg/L & $\Sigma 82$ & $64.21 \pm 58.59$ & $\Sigma 81$ & $35.62 \pm 23.89$ & $<0.001$ \\
\hline
\end{tabular}

Table 2 Logistic regression analysis of risk factors for PC 


\begin{tabular}{|c|c|c|c|c|}
\hline & \multicolumn{2}{|l|}{ Univariable analysis } & \multicolumn{2}{|c|}{ Multivariable analysis } \\
\hline & Odd ratio $(95 \% \mathrm{Cl})$ & $P$ value & Odd ratio $(95 \% \mathrm{Cl})$ & $P$ value \\
\hline Sex, male & $0.57(0.39,0.84)$ & 0.005 & & \\
\hline Age, $\geq 60$ years & $1.89(1.27,2.82)$ & 0.002 & & \\
\hline Alcohol & $2.09(1.17,3.72)$ & 0.012 & & \\
\hline Hemoglobin, $<120 \mathrm{~g} / \mathrm{L}$ & $1.91(1.23,2.97)$ & 0.004 & & \\
\hline Total bilirubin, $>17.1 \mu \mathrm{mol} / \mathrm{L}$ & $8.30(5.05,13.64)$ & $<0.001$ & $2.88(1.39,5.97)$ & 0.004 \\
\hline Alanine aminotransferase, $>50 \mathrm{U} / \mathrm{L}$ & $6.58(4.21,10.29)$ & $<0.001$ & $3.46(1.55,7.69)$ & 0.002 \\
\hline Albumin, $<35 \mathrm{~g} / \mathrm{L}$ & $1.27(0.72,2.24)$ & 0.417 & & \\
\hline Serum glucose, $>7.0 \mathrm{mmol} / \mathrm{L}$ & $2.45(1.47,4.08)$ & 0.001 & & \\
\hline Activated partial thromboplastin time, $>40 \mathrm{~s}$ & $1.21(0.68,2.14)$ & 0.521 & & \\
\hline CA199, $>25 \mathrm{U} / \mathrm{ml}$ & $18.78(11.30,31.22)$ & $<0.001$ & $10.87(5.97,19.80)$ & $<0.001$ \\
\hline Triglyceride, $>1.7 \mathrm{mmol} / \mathrm{L}$ & $1.82(1.18,2.81)$ & 0.007 & & \\
\hline Cholesterol, $>5.18 \mathrm{mmol} / \mathrm{L}$ & $2.10(1.28,3.45)$ & 0.003 & & \\
\hline High density lipoprotein, $<1.16 \mathrm{mmol} / \mathrm{L}$ & $1.74(1.13,2.67)$ & 0.012 & & \\
\hline Low density lipoprotein, $>4.14 \mathrm{mmol} / \mathrm{L}$ & $3.10(1.25,7.69)$ & 0.015 & & \\
\hline
\end{tabular}

Table 3 Clinicopathological and follow-up information of PC patients in different lipid level

\begin{tabular}{|c|c|c|c|c|c|c|c|c|c|c|}
\hline & High CHOL & Low CHOL & $\begin{array}{l}\mathrm{P} \\
\text { value }\end{array}$ & High TG & Low TG & $\begin{array}{l}\mathrm{P} \\
\text { value }\end{array}$ & Low HDL & High HDL & $\begin{array}{l}P \\
\text { value }\end{array}$ & High LDL \\
\hline \multirow{2}{*}{$\begin{array}{l}\text { Tumor size, } \\
\mathrm{cm}\end{array}$} & 73 & 164 & $<0.001$ & 96 & 141 & 0.057 & 158 & 69 & 0.869 & 27 \\
\hline & $2.91 \pm 1.04$ & $3.63 \pm 1.48$ & & $3.20 \pm 1.24$ & $3.55 \pm 1.47$ & & $3.37 \pm 1.41$ & $3.40 \pm 1.38$ & & $3.50 \pm 1.40$ \\
\hline $\begin{array}{l}\text { Operation } \\
\text { type }\end{array}$ & 73 & 169 & 0.143 & 97 & 145 & 0.102 & 162 & 69 & 0.124 & 27 \\
\hline $\begin{array}{l}\text { Radical } \\
\text { resection }\end{array}$ & 71 (97.3\%) & $156(92.3 \%)$ & & 94 (96.9\%) & $133(91.7 \%)$ & & $152(93.8 \%)$ & 68 (98.6\%) & & 27 (100.0\%) \\
\hline $\begin{array}{l}\text { Palliative } \\
\text { resection }\end{array}$ & $2(2.7 \%)$ & $13(7.7 \%)$ & & $3(3.1 \%)$ & $12(8.3 \%)$ & & $10(6.2 \%)$ & $1(1.4 \%)$ & & $0(0.0 \%)$ \\
\hline AJCC stage & 73 & 169 & 0.055 & 97 & 145 & 0.074 & 162 & 69 & 0.246 & 27 \\
\hline stage IㅁII & $56(76.7 \%)$ & $113(66.9 \%)$ & & 74 (76.3\%) & 95 (65.5\%) & & 118 (72.8\%) & 45 (65.2\%) & & $20(74.1 \%)$ \\
\hline stage IIIIIV & $17(23.3 \%)$ & $56(33.1 \%)$ & & $23(23.7 \%)$ & 50 (34.5\%) & & 44 (27.2\%) & 24 (34.8\%) & & 7 (25.9\%) \\
\hline $\begin{array}{l}\text { Differentiation } \\
\text { degree }\end{array}$ & 67 & 143 & 0.601 & 86 & 128 & 0.394 & 146 & 62 & 0.186 & 25 \\
\hline Well & $13(19.4 \%)$ & 27 (18.9\%) & & 15 (17.4\%) & 25 (19.5\%) & & 27 (18.5\%) & 12 (19.4\%) & & $7(28.0 \%)$ \\
\hline Moderate & $36(53.7 \%)$ & 71 (49.7\%) & & 41 (47.7\%) & $66(51.6 \%)$ & & $68(46.6 \%)$ & $36(58.1 \%)$ & & $11(44.0 \%)$ \\
\hline Poor & $18(26.9 \%)$ & $45(31.5 \%)$ & & 30 (34.9\%) & 37 (28.9\%) & & 51 (34.9\%) & $14(22.6 \%)$ & & 7 (28.0\%) \\
\hline \multirow{2}{*}{$\begin{array}{l}\text { Nerve } \\
\text { invasion }\end{array}$} & 67 & 148 & 0.574 & 89 & 126 & 0.664 & 142 & 66 & 0.977 & 26 \\
\hline & 37 (55.2\%) & $80(54.1 \%)$ & & 50 (56.2\%) & $67(53.2 \%)$ & & 75 (52.8\%) & 35 (53.0\%) & & $13(50.0 \%)$ \\
\hline \multirow{2}{*}{$\begin{array}{l}\text { Vascular } \\
\text { invasion }\end{array}$} & 68 & 148 & 0.874 & 90 & 126 & 0.977 & 143 & 66 & 0.140 & 26 \\
\hline & $12(17.6 \%)$ & 31 (20.9\%) & & $18(20.0 \%)$ & 25 (19.8\%) & & 32 (22.4\%) & $9(13.6 \%)$ & & $3(11.5 \%)$ \\
\hline \multirow{2}{*}{$\begin{array}{l}\text { Follow-up, } \\
\text { months }\end{array}$} & 73 & 169 & 0.083 & 97 & 145 & 0.846 & 162 & 69 & 0.065 & 27 \\
\hline & $80.99 \pm 69.65$ & $64.86 \pm 64.57$ & & $70.74 \pm 63.92$ & $69.04 \pm 68.24$ & & $66.57 \pm 62.13$ & $84.38 \pm 76.74$ & & $102.67 \pm 67.72$ \\
\hline \multirow{2}{*}{$\begin{array}{l}\text { Disease } \\
\text { related death }\end{array}$} & 73 & 169 & 0.959 & 97 & 145 & 0.774 & 162 & 69 & 0.397 & 27 \\
\hline & 40 (54.8\%) & 92 (54.4\%) & & 54 (55.7\%) & 78 (53.8\%) & & 92 (56.8\%) & 35 (50.7\%) & & $15(55.6 \%)$ \\
\hline
\end{tabular}




\section{Figures}

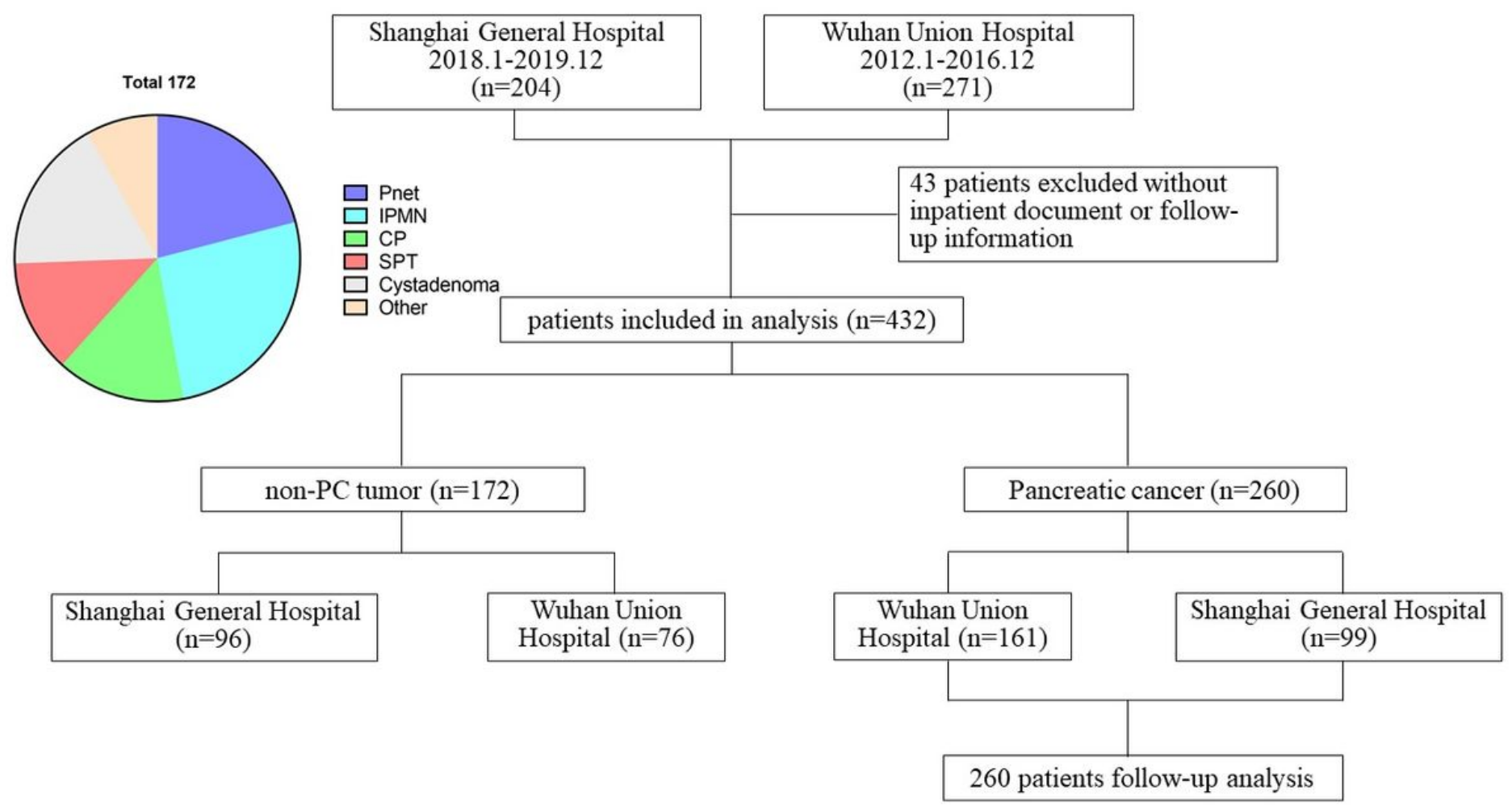

Figure 1

Schematic diagram regarding patient data. 


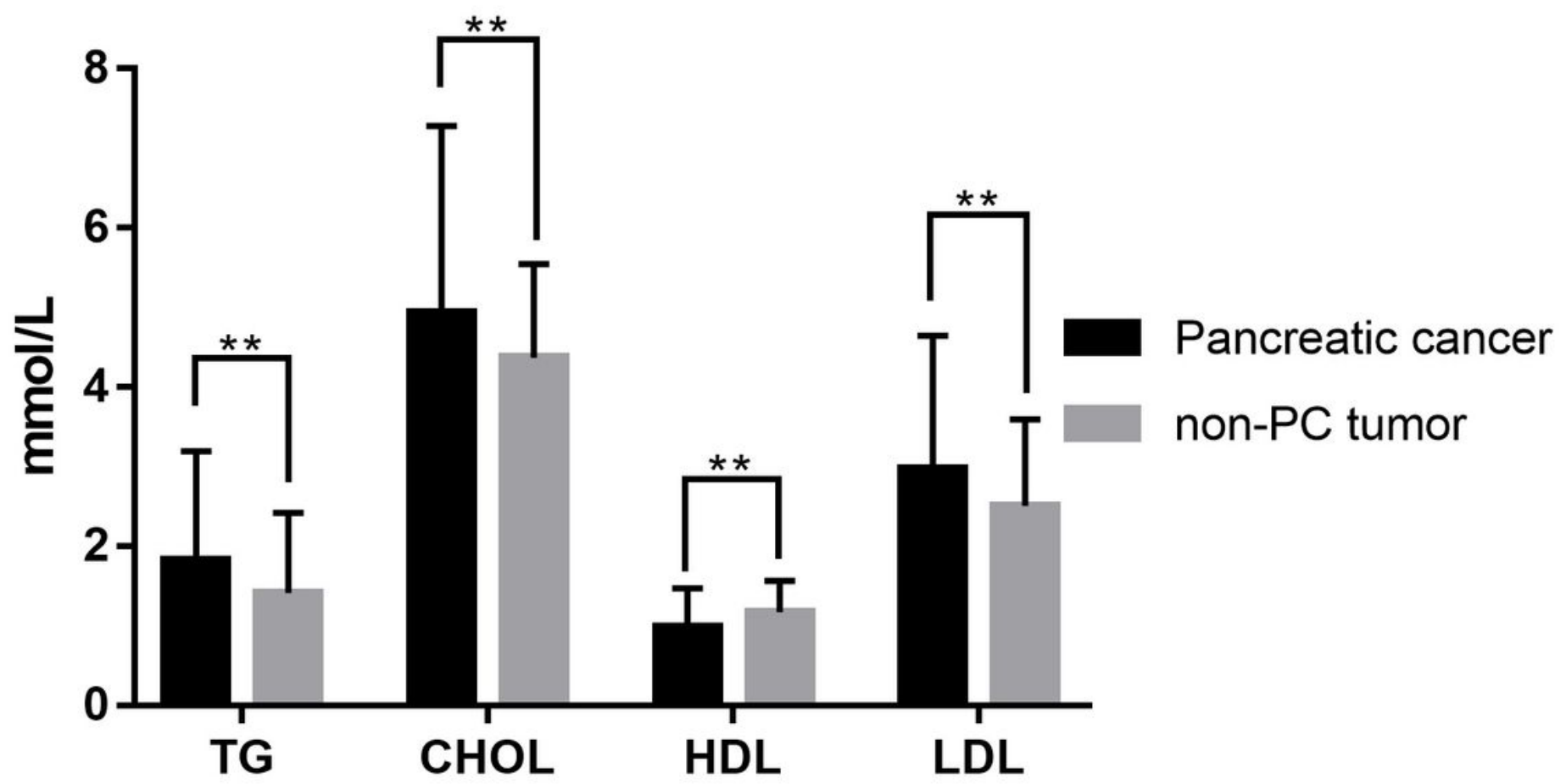

Figure 2

Serum lipid difference between PC and non-PC tumor patients in our cohort. ** , $P<0.05$.
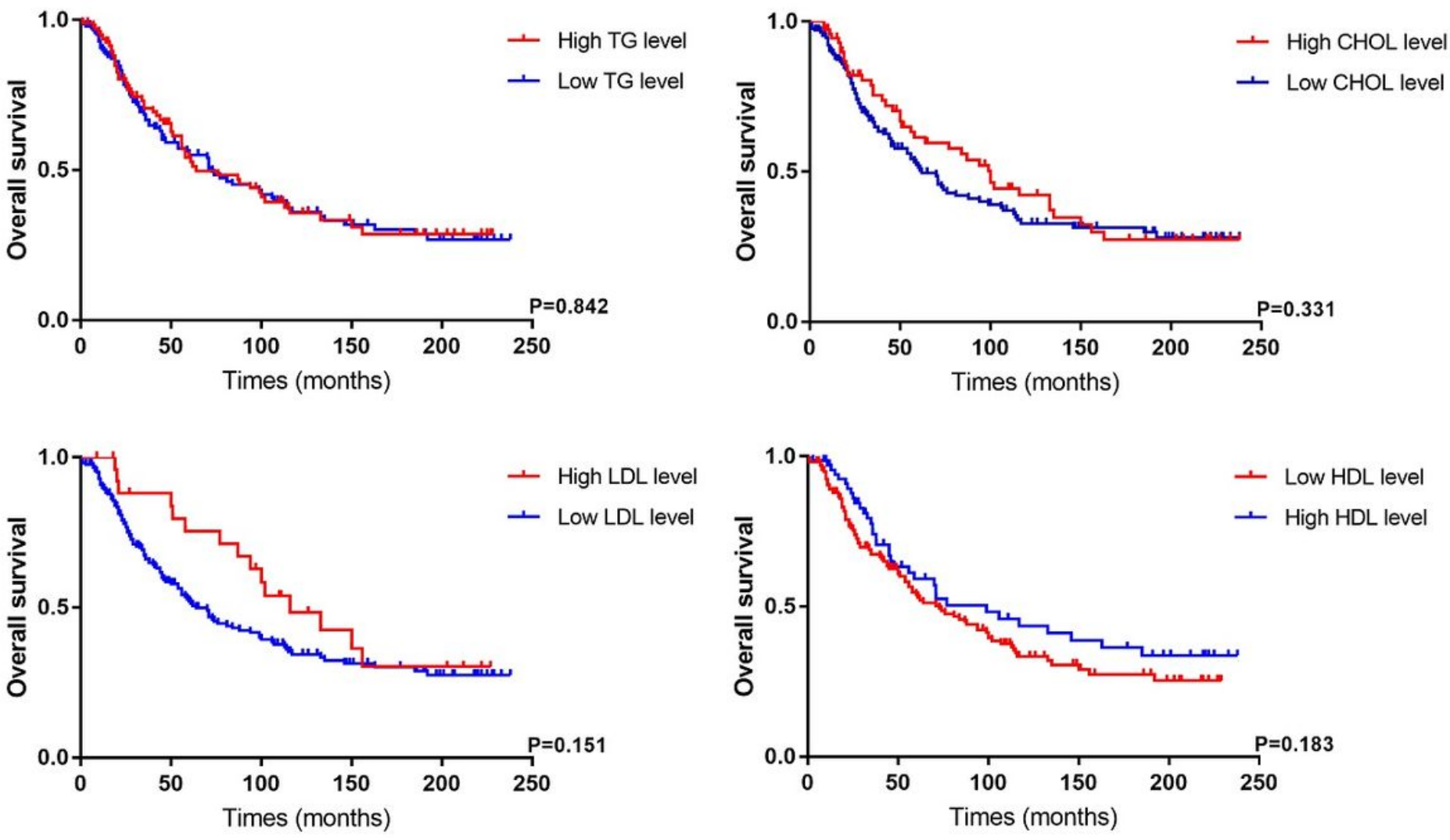

Figure 3

Survival analysis of PC patients according to different lipid level in our cohort. 


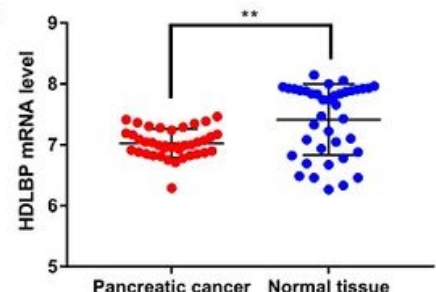

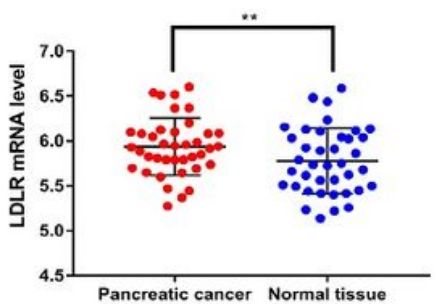

b
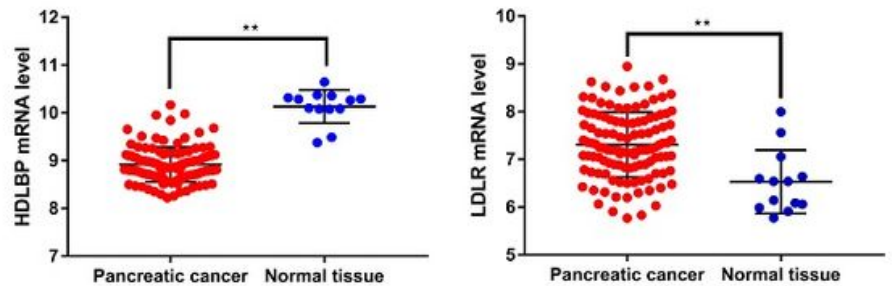

C

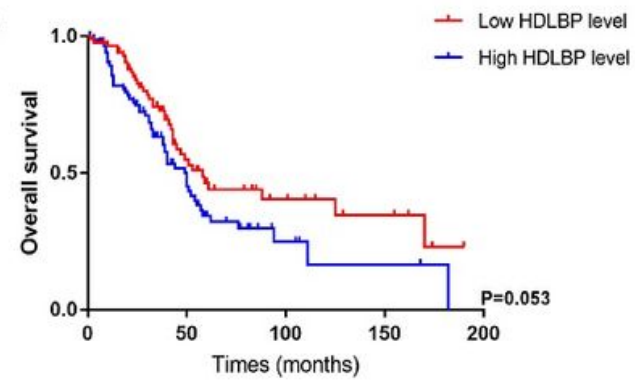

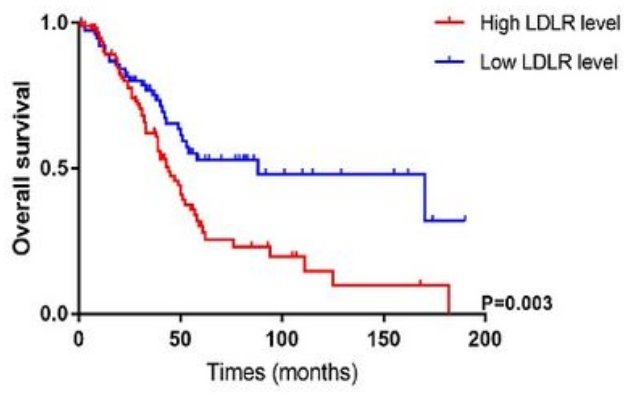

Figure 4

Differential and survival analysis of related lipoprotein from database data. $a$ and $b$, The expression level of HDLBP and LDLR in PC tissue and paracancerous normal tissue from GSE15471 and GSE62165, ** P<0.05. c TCGA database was searched to analyze overall survival of PC patients in different HDLBP and LDLR expression level.

\section{Supplementary Files}

This is a list of supplementary files associated with this preprint. Click to download.

- supplmentarymaterial.doc 